\title{
Huriet Law
}

National Cancer Institute

\section{Source}

National Cancer Institute. Huriet Law. NCI Thesaurus. Code C142572.

A French regulation covering the initiation and conduct of all interventional clinical studies,

which seeks to protect the participants within interventional trials as well as those individuals and entities that sponsor the trial. 